\title{
The Application of Electrical Automation in Electrical Engineering
}

\author{
Zhang Pingli ${ }^{l}$, Fang Yonghao ${ }^{2}$, Fang $\mathrm{Fei}^{3}$
}

1. Puyang Municipal Facilities Management Office, Puyang, Henan,457000, China

2. No. 1 Senior Middle School, Puyang, Henan, 457000, China

3. Maichi Technology Co., Ltd., Puyang, Henan, 457000, China

Abstract: To apply automation in electrical engineering is to use a series of devices with functions of automatic detection and control to realize the realtime or remote control, management, and monitoring of the original electrical system. Therefore, the electrical automation technology has broad development space in the construction of electrical engineering, and it is worth of application and study for relevant electrical installation engineering and scientific research departments. In the actual production process, it is necessary to optimize the production mode, reduce the cost, and keep the good working performance of electrical equipment. Based on this, this article briefly analyzes the application of electrical automation in electrical engineering, hoping to provide an effective reference.

Key words: Electrical automation; electrical engineering; application

\section{Basic Concepts of Electrical Automation De- sign}

\subsection{Design Concept of Centralization}

In terms of electrical engineering, the same processor is responsible for the centralized process of all system functions. Therefore, the processor itself is under enormous pressure and burden, which has an impact on its efficiency. And the service objects of the monitoring of electrical equipment are so many that the host has to bear a great burden, so more cables are needed, which means the increase of investment costs. In addition, the introduction of long-distance cable will directly affect the reliability and stability of the power system. When it operates under high load for a long time, a malfunction is more likely to happen.
Therefore, the concept of centralized monitoring is the most commonly used in the application of automation technology in electrical engineering.

\subsection{Design Concept of Remoting}

The application of remoting can not only reduce the use of cable, but also save more investment costs, and significantly improve the security and stability. However, it also increases electrical communication, so this design is often used in smaller electrical engineering.

\subsection{Design Concept of Field Bus}

With the arrival of the information age, network technology and computer technology are used more and more widely. Field bus is the representative of network technology, the application of which can significantly enhance the targeted design of the system. And it can adopt different functions according to the corresponding interval, thus meeting the actual requirements. Based on the advantages of remoting, the concept of field bus can reduce equipment spacing, analog quantity, and terminal cabinet. Therefore, this method is widely used in the field of electrical engineering, and will become the trend of the development of electrical automation in the future.

\section{Advantages of Applying Electrical Automa- tion to Electrical Engineering}

\subsection{It helps to Speed up Intelligent Development of Electrical Engineering}

In order to fully meet the requirements of the reform 
of market economic system and enlarge the scope of services in the application of electrical engineering, it is necessary to promote its intelligent development and gradually realize the intellectualization of electrical equipment, which requires to apply electrical automation to electrical engineering and speed up the intelligent development of electrical engineering. More specifically, (1) The electrical engineering based on electrical automation can let relevant enterprises form an intelligent management mode accordant with their own development needs in the practice process; (2) The electrical engineering based on electrical automation has obvious advantages in intelligent development, which is helpful to improve the intelligence level in the production process; (3) Through the interaction with the computer network, the electrical engineering based on electrical automation is conducive to the construction of information management system, and enables itself to be in a stable state of development.

\subsection{It Helps to Perfect the Monitoring Function of Electrical Engineering}

In the course of electrical engineering practice, the emphasis on the introduction of electrical automation and related technologies can continuously improve the monitoring level in the service area, and perfect the monitoring function in the future development. More specifically, (1) The actual working condition of the electrical equipment in the electrical engineering based on electrical automation can be monitored in real time, which helps to reduce the occurrence rate of malfunctions; (2) The information produced by each production link of the electrical engineering based on electrical automation can be obtained in time, which ensures the safety implementation of production plan, and continuously improves the monitoring level in the engineering practice; (3) The electrical engineering based on electrical automation will form an effective monitoring mode to realize the timely elimination of the potential safety hazards.

\subsection{It Helps to Promote the Production Level of Electrical Engineering Practice}

In the development of electrical automation, the integration of the advantages of information technology and computer network is significant to the improvement of the production level of electrical engineering practice. More specifically, (1) The equipment performance of the electrical engineering supported by electrical automation can be optimized constantly, thus enhancing the working stability of electrical equipment and the production level of electrical engineering practice; (2) The production mode of the electrical engineering supported by electrical automation can be optimized to provide scientific guidance for the safety implementation of production plan, and constantly promote its production level; (3) In the production practice of the electrical engineering supported by electrical automation, the occurrence rate of problems is reduced, which ensures an excellent production level of electrical engineering.

\subsection{Protective Function}

The operation of electric power system is affected by various factors, such as the complexity of environment, the diversification of equipment, or the nonstandard connection of power supply line. Thus the equipment malfunction and the circuit fault are likely to happen. It's difficult for traditional manual monitoring and overhaul to fully grasp the operating state of the equipment, which leads to various potential safety hazards. When the equipment breaks down or the circuit is unstable, the electrical automation control technology can cut off the power safely, terminate the operational procedure, avoid safety accidents and economic losses, guarantee the safe operation of electrical equipment.

\section{Application of Electrical Automation Tech- nology in Electrical Engineering}

\subsection{Application of PLC Technology}

PLC technology means to control working links mainly through simple programming. The requirements of programming are not high. As long as knowing the objects and the requirements of control, ordinary technicians can achieve controlling through programming. The outstanding features of PLC technology are convenient use, simple program, strong functions, adaptation to different working environments, and lower cost than other similar technologies. This shows that PLC technology has low requirements and a wide range of applications. Therefore, it is widely used in different working environments. Electrical automation replaces the original controller with PLC technology so as to reduce the cost, enhance the efficiency, and realize the high efficient and high quality work of electrical automation.

In the process of using PLC technology, it's necessary to pay attention to the programming. The programme is the key factor that makes PLC techno- 
logy play its own role. Anything wrong with the programme will seriously affect electrical automation, even the stable operation of electrical engineering.

\subsection{Monitoring of Electrical Automation Tech- nology}

The monitoring of electrical automation in electrical engineering is concerned with whether electrical automation control technology can be widely used at work, which is the focus of our study. In conventional substations, transformers and disconnectors are the two most critical hardware. If they break down at work, the electrical equipment can not run normally. Therefore, traditional detection requires to spend time and manpower for real-time monitoring so as to deal with any emergency situation in time and ensure the normal operation of the equipment. The electrical system now has a microcomputer as its technical basis, which is the advantage of automatic electrical equipment. This technology can realize the real-time monitoring of the changes of transformers and disconnectors to make sure they run normally all the time. Microcomputers, of course, is not only used in electrical systems. It can also effectively analyze the problems exiting in other production areas and the direction of their future development. Therefore, the great advantages of electrical automation control technology should be given full play in entire electrical engineering, and the monitoring level of electrical automation needs to be improved effectively, which is the trend of future development. In other words, the electrical automation control equipment plays a significant role in the intelligent development and reform of electrical engineering, and it has laid a solid foundation for the innovation of intelligent technologies.

\subsection{Intelligent Application of Electrical Automation Technology}

In the future, the electrical automation in electrical engineering will develop towards the direction of intelligent technologies, bringing great convenience to both life and work. In electrical engineering, the secondary equipment of the electrical system has a lot of requirements for the time and space of installation. There should be an appropriate distance between the primary and secondary equipment. Those two are connected through control cable and power cable. In order to greatly save power cable and control cable, the function of the conventional secondary equipment must be considered when the structure of the primary equipment is designed. Intelligent application is an important reform of electrical engineering. Electrical engineering and electrical automation have been gradually applied to various industries and fields in recent years, becoming an important subject in the field of electrical application. Closely related to people's daily life and the social industry, it has gradually developed into an important branch of high and new technology, playing an irreplaceable role in the development of national economy.

\subsection{Application in Energy Conservation and Moni- toring in Power Plants}

Whether the energy-saving and monitoring problems in electrical engineering construction can be dealt with effectively is related to the production cost and benefit. The effective results of energy saving and monitoring in power plants involved in the development of electrical engineering require to strengthen the use of electrical automation. More specifically, (1) The introduction of electrical auto-mation can analyze the energy consumption status of the equipment and circuit of the power plant, and then work out an effective working plan to make sure the energy-saving idea can be reflected throughout the production practice of the power plant, so as to keep good environmental protection benefit on the basis of increasing its economic benefit, and ensure the steady development of the power plant. (2) Based on power plant's actual requirements for decentralized monitoring in the production process, the introduction of electrical automation can perfect the service function of the monitoring system, enhance the control effect in the running process of the system, realize the integrated use of monitoring information and the realtime monitoring of the power plant's production process. In addition, the monitoring equipment based on electrical automation can obtain the operation information of the production equipment of the power plant, so as to provide convenience for its maintenance work.

\subsection{Application in Power Grid Dispatching}

The power grid dispatching system consists of the point channel of the automatic power plant, the station end and the control center. The main components involved are power grid dispatching workstation, large screen display and central server. Power plant, power grid dispatching center and terminal substation can be connected together through the local area network of the power system, so that the monitoring work can be carried out. In addition, the automation 
technology applied in electrical engineering can monitor the working condition of the power grid at any time, so as to grasp the real-time information, and further confirm whether the power system can load the current power consumption on the basis of data analysis, which realizes the real automatic control of the power grid dispatching. Therefore, it is necessary to strengthen the use of electrical automation and related technologies, improve the dispatching level, and ensure the stability of the power system.

\subsection{Application in Substation}

The application of electrical automation technology in electrical engineering also plays an important role in the substation. It can combine electronic information technology and automation and control technology through electronic computers, and form an automated information system through transmission technology. The electrical automation system is applied mainly in the operation management of the substation. It replaces the tediousness of manual operation with its intellectualization, improves the underdeveloped production condition, enhances the production efficiency and ensures the safety of production.

\subsection{Application in Distribution System}

Electrical automation plays an important role in distribution system. and its application scale needs to be enlarged to promote the positive effect of electrical automation. At present, the application of electrical automation in distribution system is embodied in the following aspects: distribution automation with centralized monitoring, distribution automation with the combination of centralized monitoring and distribution management, and distribution automation with local control, of which the first two modes are more commonly used. Both of them adopt the distributed structure, realize the unification of time and the effective connection of substation and master station, establish the highly-centralized distribution automation system. The application of electrical automation in distribution system can scientifically and reasonably plan power information, carry out efficient and accurate distribution work, improve the reliability of power supply and the efficiency of the whole electrical engineering.

\section{Direction of Electrical Automation's Future Development}

\subsection{Opening}

As an inevitable trend, opening is a core technology that can be used to achieve dispatching automation. With the network as the carrier, it can also help to connect the electrical automation structure and the external interface, so as to form the power system movement technology. In the aspects of equipment design and manufacturing, electrical automation technology can integrate the advantages of computers and simulation technology, establish an open technology structure, and meet the application needs of different fields, so that information technology can provide technical supports for the development of electrical automation, and play its practical value.

\subsection{Generalization}

The application of electrical automation in network structure can ensure the ordered exchange of hardware and software product data between manufacturers, deal with the problems faced in the practice, and guarantee the smooth running of production work. As for computer operation, electrical automation system can realize the automatic control and management through hardware, make sure that the data information can be shared in real time, reduce and eliminate the asymmetric information. It can not only improve the efficiency of data exchange, but also save more cost.

\subsection{Creativeness}

At present, there are many electrical automation products on the market. The enterprises must carry out production strictly in accordance with national standards and requirements. In order to stand out from the intense market competition, they should continue to innovate, increase the content of science and technology in their products, enhance their comprehensive competitiveness. China has introduced many policies to encourage and guide enterprises to devote more time and energy to product innovation. The enterprises are also supported to actively introduce more advanced technologies and optimize the performance of existing electrical automation products, so that the value of the products can be given full play on the market.

\subsection{Security}

With the rapid development of the national economy, people's living standard has improved significantly. They are paying more attention to their own security needs, which puts forward corresponding requireme- 
nts for the safety and reliability of products. Therefore, security technology and safety control technology are used in the production of electrical automation products to ensure the security of users in unsecured environments and avoid the threats for economic property. Moreover, after continuous improvement, the electrical automation safety system has ensured the safety production of electrical automation, and injected more vitality to its future development.

\subsection{Intellectualization}

The wide application of electrical automation control system has brought great convenience to people's life and work. At present, with the increase of Ethernet transmission rate, the electrical automation control system is confronted with greater challenges and opportunities. Therefore, in order to ensure the sustainable development of the electrical automation control system, relevant researchers should pay attention to the research on the electrical automation control system, and speed up its intelligent development, so as to meet the development demands of the current market. In addition, many PLC manufacturers are studying and developing the fault detection intelligent module, which reduces the probability of equipment malfunction and increases the reliability and security of the system. In short, many automation control manufacturers begin to realize the importance of automation control technology, which promotes the intelligent development of electrical automation control, and has laid a solid foundation for the continuous development of China's social economy.

\subsection{Energy Conservation}

The energy-saving design of electrical engineering needs to be popularized and applied in the development and construction of enterprises, so that the pollution discharge of enterprises can be reduced and the energy can be saved. This can promote the sustainable development of electric power enterprises and have great significance for the realization of harmonious coexistence between man and nature. Energy conservation in industrial electrical automation has four development trends:

(1) Energy conservation in distribution line

In the construction of the plants, the key task of the distribution line is to transport power to the facilities. In this process, the distribution line will consume some electrical energy. Although compared with the power consumption of the entire system, it seems to be insignificant, after accumulation, its consumption of power can not be ignored. Resistance is the main reason for the loss of the power in the distribution line. In order to reduce the energy consumption in the transmission process, it's necessary to first reduce the resistance of the distribution line, especially when the power consumption and load capacity of the distribution line are determined.

(2) Energy conservation in reactive power compensating system

When power distribution involves industrial enterprises, the inductive load such as transformers and motors should be reasonable and scientific to improve the power factor of the part which occupies a large proportion of power consumption. Or use the method of artificial compensation of reactive power to realize the efficiency of power supply and distribution system. This method is a compensating device for increasing the reactive power of the system as well as Factor elevation of facility power, and the method of artificial reactive compensation according to the reactive energy consumption will change depending on conditions.

\section{(3) Energy conservation in illuminating system}

Illuminating system requires big electricity consumption, so the energy-saving plan of power industries and enterprises must include the production of it. The design of illuminating system must be based on the design specification. The design strategy should be determined after the careful and rigorous calculation of the power density value and illuminating value of illuminations in various production sites. The lighting power value and illuminating brightness should be examined and calculated earnestly according to the current illuminations operation requirements of our country, so as to realize the effect of energy conservation.

(4) Energy conservation in light-current system Industrial production is carried out in the industrial factory buildings, so it's necessary to improve the safety and fire protection design of the plants. The fire risk of the industrial factory buildings must be reduced to guarantee the safety production of industrial enterprises. Various security defense systems should be installed to supervise and manage the safety of the plants, such as access control system, monitoring system, or intrusion prevention system. Although the use of light-current system in the plants can ensure the production safety, the energy consumption of electrical devices increases. Therefore, the design of the light-current system should be strengthened to reduce the power consumption.

In conclusion, the electrical automation technology has a relatively good application effect in electrical 
engineering. It can promote the intelligent development of electrical system. Therefore, based on the actual situation, we need to study hard and innovate actively, broaden our way of thinking, make contributions to the application and development of electrical automation in electrical engineering.

\section{References}

[1] $\mathrm{Xu} \mathrm{Xu}$, Tong Lingling. Analysis on the Application of Electrical Automation in Electrical Engin- eering $[\mathrm{J}]$.Construction Materials and Decoration, 2016(53):151-152.

[2] Geng fushun. On the Application of Electrical Automation Technology in Electrical Engineering[J]. Heilongjiang Science and Technology Information, 2016(36):12.

[3] Cai Jing. Discussion on the Automation Technology in Electrical Engineering[J]. Heilongjiang Science and Technology Information, 2016(36):26. 